\title{
Leapfrogging vortex rings: Hamiltonian structure, geometric phases and discrete reduction
}

\author{
Banavara N. Shashikanth ${ }^{\mathrm{a}, *}$, Jerrold E. Marsden ${ }^{\mathrm{b}}$ \\ ${ }^{a}$ Department of Mechanical Engineering, New Mexico State University, Las Cruces, NM 88003, USA \\ ${ }^{\mathrm{b}}$ Control and Dynamical Systems 107-81, California Institute of Technology, Pasadena, CA 91125-8100, USA
}

Received 12 August 2002; accepted 2 May 2003

Communicated by E. Knobloch

\begin{abstract}
We present two interesting features of vortex rings in incompressible, Newtonian fluids that involve their Hamiltonian structure.

The first feature is for the Hamiltonian model of dynamically interacting thin-cored, coaxial, circular vortex rings described, for example, in the works of Dyson (Philos. Trans. Roy. Soc. London Ser. A 184 (1893) 1041 ) and Hicks (Proc. Roy. Soc. London Ser. A 102 (1922) 111). For this model, the symplectic reduced space associated with the translational symmetry is constructed. Using this construction, it is shown that for periodic motions on this reduced space, the reconstructed dynamics on the momentum level set can be split into a dynamic phase and a geometric phase. This splitting is done relative to a cotangent bundle connection defined for abelian isotropy symmetry groups. In this setting, the translational motion of leapfrogging vortex pairs is interpreted as the total phase, which has a dynamic and a geometric component.

Second, it is shown that if the rings are modeled as coaxial circular filaments, their dynamics and Hamiltonian structure is derivable from a more general Hamiltonian model for $N$ interacting filament rings of arbitrary shape in $\mathbb{R}^{3}$, where the mutual interaction is governed by the Biot-Savart law for filaments and the self-interaction is determined by the local induction approximation. The derivation is done using the fixed point set for the action of the group of rotations about the axis of symmetry using methods of discrete reduction theory.
\end{abstract}

(c) 2003 Published by The Japan Society of Fluid Mechanics and Elsevier B.V. All rights reserved.

PACS: $11.15 . \mathrm{E}, 47.15 . \mathrm{K}, 05.45,02.20 . \mathrm{T}$

Keywords: Axisymmetric vortex rings; Leapfrogging motions; Geometric mechanics; Hamiltonian systems; Symmetry reduction; Geometric phases

\footnotetext{
${ }^{*}$ Corresponding author. Tel.: 505-646-4348; fax: 505-646-6111.

E-mail addresses: shashi@me.nmsu.edu (B.N. Shashikanth), marsden@cds.caltech.edu (J.E. Marsden).
}

0169-5983/\$30.00 (c) 2003 Published by The Japan Society of Fluid Mechanics and Elsevier B.V.

All rights reserved.

doi:10.1016/j.fluiddyn.2003.05.001 


\section{Introduction}

The use of geometric analysis methods in fluid mechanics has now become rather common. See, for example, the books by Arnold and Khesin (1998) and Marsden and Ratiu (1999) and references therein. In this paper we apply some of these techniques to the problem of leapfrogging vortex rings. See Saffman (1992) for a general introduction to the dynamics of vortex rings.

The Hamiltonian structure of vortex filaments and other special fluid motions was given in Marsden and Weinstein (1983). This structure has several interesting uses, such as in understanding the links to the nonlinear Schrödinger integrable structure (see, for example Langer and Perline, 1991), stability questions, etc. Meanwhile, geometric phases have also been developed, both for traditional mechanical systems, as in Marsden et al. (1990) as well as in fluid problems involving vortices, as in Shashikanth and Newton (1998).

A different model of dynamically interacting coaxial circular vortex rings with circular cores of small but finite radius was studied, among others, by Dyson (1893) and Hicks (1922). This model, which does not use a self-induction approximation as in the filament models, but rather has a core structure, also has an interesting Hamiltonian structure. See Lamb (1932) for a list of historical references. Unfortunately, this theory is restricted to the case of coaxial circular rings with circular cores of small radius.

The phenomenon of leapfrogging using this model was studied in detail by Hicks (1922), though the first mention of this phenomenon in the literature goes back earlier (see, for instance Love, 1894). The reader is again referred to Lamb (1932). Movies and photographs of leapfrogging vortex rings in an experimental setting may be found on the web page of Lim (1997).

The purpose of this paper is to apply Hamiltonian structures and symplectic reduction (both continuous and discrete) as well as the theory of geometric phases to the classical problem of leapfrogging circular vortex rings in axisymmetric flows. We shall see that the translational motion of the rings can be interpreted as a reconstruction phase in an interesting way. The phase is obtained by reconstructing a periodic motion on the symplectic reduced space, where the reduction is done via translational symmetries. The phase is made up of a dynamic and geometric part and the geometric phase has an interesting interpretation in terms of the area of the region enclosed by the reduced periodic trajectory. This is consistent with the fact that the leapfrogging motion is periodic modulo translational symmetries.

We also show that if the vortex rings are modeled as filaments (i.e. as curves, without cores) the dynamics and Hamiltonian structure of circular filament rings fits into the general problem of interacting vortex filament rings of arbitrary shape using the ideas of discrete symmetries and fixed point subspaces.

The geometric phase analysis is done for the Dyson-Hicks model of rings with thin cores, but a similar analysis applies to the case of circular filament rings.

\section{Symplectic structure of $N$ translating, circular vortex rings}

\subsection{Equations of motion}

We consider the equations of evolution of $N$ thin-cored circular vortex rings aligned along a fixed axis. Let the radius of the $i$ th ring be denoted $R_{i}$ and lets its distance from a reference point on the 
common axis of symmetry be denoted $X_{i}$. The equations of motion for these rings are given by (see Dyson, 1893; Hicks, 1922; Konstantinov, 1994):

$$
\begin{aligned}
\frac{\mathrm{d} X_{i}}{\mathrm{~d} t} & =\frac{\Gamma_{i}}{4 \pi R_{i}}\left(\log \frac{8 R_{i}}{a_{i}}-\frac{1}{4}\right)+\frac{1}{\Gamma_{i} R_{i}} \frac{\partial U}{\partial R_{i}}, \\
\frac{\mathrm{d} R_{i}}{\mathrm{~d} t} & =-\frac{1}{\Gamma_{i} R_{i}} \frac{\partial U}{\partial X_{i}}, \quad i=1, \ldots, N,
\end{aligned}
$$

where

$$
U=\frac{1}{\pi} \sum_{i=1}^{N} \sum_{j>i}^{N} \Gamma_{i} \Gamma_{j} I_{i j}
$$

and

$$
I_{i j}=\int_{0}^{\pi} \frac{R_{i} R_{j} \cos \theta}{\sqrt{\left(X_{i}-X_{j}\right)^{2}+R_{i}^{2}+R_{j}^{2}-2 R_{i} R_{j} \cos \theta}} \mathrm{d} \theta .
$$

In these equations of motion, $\Gamma_{i}$ is the vortex strength of the $i$ th ring (a constant) and $a_{i}$ is the radius of the thin core, which is assumed to have a circular shape throughout the motion. The core radius is not a free parameter but is fixed by the radius of the ring at any instant by the relation:

$$
a_{i}(t)^{2} R_{i}(t)=A_{i}=\text { constant. }
$$

This relation follows from the vorticity evolution equation (in cylindrical polar coordinates $(r, \theta, z)$ ) for axisymmetric swirl-free flow (see Saffman, 1992), namely:

$$
\frac{\mathrm{D}}{\mathrm{D} t}\left(\frac{\omega_{\theta}}{r}\right)=0
$$

and the conservation of the strength, $\Gamma_{i}:=\int_{i} \omega_{\theta} \mathrm{d} r \mathrm{~d} z$, of each ring. The term $I_{i j}$ can be expressed in terms of elliptic integrals of the first and second kind; see cited references above.

\subsubsection{Hamiltonian structure}

Define $p_{i}=\Gamma_{i} R_{i}^{2}$ and consider the canonical symplectic two-form:

$$
\Omega=\sum \mathrm{d} X_{i} \wedge \mathrm{d} p_{i}
$$

on the phase space $P=\left(\mathbb{R}^{N} \times\left(\mathbb{R}^{N}\right)^{*}\right) \backslash \Delta \equiv \mathbb{R}^{2 N} \backslash \Delta$, where $\Delta$ is the set of collision points defined by

$$
\Delta:=\left\{\left(X_{j}, X_{k}, p_{j}, p_{k}\right) \mid X_{j}=X_{k}, R_{j}=R_{k}, j \neq k\right\} .
$$

Define the Hamiltonian function $H: P \rightarrow \mathbb{R}$ by

$$
H\left(X_{i}, p_{i}\right)=\sum_{i=1}^{N} \frac{\Gamma_{i}^{3 / 2} p_{i}^{1 / 2}}{2 \pi}\left(\log \left\{\frac{8}{A_{i}}\left(\frac{p_{i}}{\Gamma_{i}}\right)^{3 / 4}\right\}-\frac{7}{4}\right)+2 U .
$$

It is easy to check that the system of Eqs. (2.1) and (2.2) is Hamiltonian with respect to the symplectic form $\Omega$ defined in (2.3) and the Hamiltonian $H$ defined by (2.4). That is, under the 


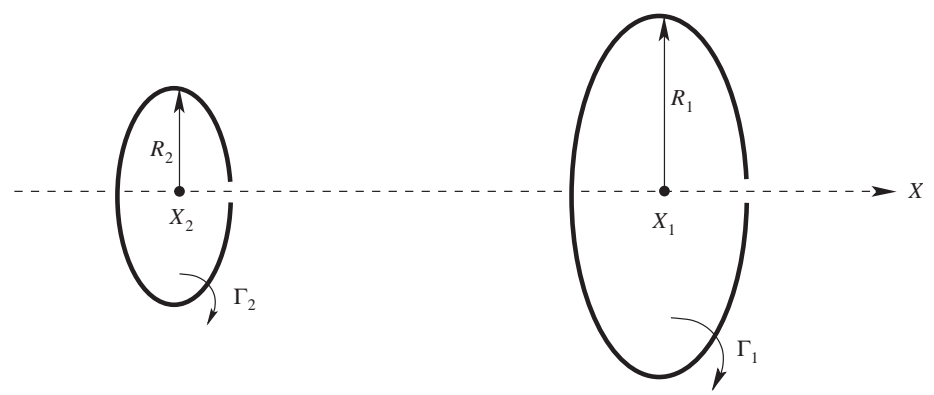

Fig. 1. Two interacting coaxial circular vortex rings, under certain initial conditions, exhibit a periodic interpenetrating motion called "leapfrogging". The smaller one accelerates towards the larger one, passes through it, becomes the larger one and the process repeats.

change of variables $p_{i}=\Gamma_{i} R_{i}^{2}$, these equations are equivalent to the system

$$
\begin{aligned}
\frac{\mathrm{d} X_{i}}{\mathrm{~d} t} & =\frac{\partial H}{\partial p_{i}}, \\
\frac{\mathrm{d} p_{i}}{\mathrm{~d} t} & =-\frac{\partial H}{\partial X_{i}} .
\end{aligned}
$$

For $N=2$, as shown in Fig. 1, the well-known phenomenon of leapfrogging may occur for certain choices of initial conditions and parameters (see Hicks, 1922; Gurzhii et al., 1988).

\subsection{A connection on $P$ and the associated geometric phase}

For the basic background on geometric mechanics that is used in the following, we refer to Marsden and Ratiu (1999) and Marsden (1992).

\subsubsection{The reduction bundle}

Consider a cotangent bundle $T^{*} Q$ with its canonical symplectic structure on which a symmetry group $G$ acts by the cotangent lift of a free, proper, smooth action of $G$ on $Q$. In this situation, there is a naturally associated reduction bundle $\pi_{\mu}: \mathbf{J}^{-1}(\mu) \rightarrow P_{\mu}$, where $\mathbf{J}$ is the natural equivariant momentum map for the action, $\mathbf{J}^{-1}(\mu)$ is the $\mu$-level set of the momentum map and $P_{\mu}=\mathbf{J}^{-1}(\mu) / G_{\mu}$ is the symplectic reduced space. Here, $G_{\mu}$ is the isotropy group of the point $\mu$, namely, the subgroup of $G$ leaving $\mu$ pointwise fixed under the coadjoint action of $G$ on $\mathfrak{g}^{*}$, the dual of the Lie algebra of $G$. Recall that the momentum map $\mathbf{J}: T^{*} Q \rightarrow \mathfrak{g}^{*}$ for cotangent lifted actions is given by

$$
\left\langle\mathbf{J}\left(\alpha_{q}\right), \xi\right\rangle=\left\langle\alpha_{q}, \xi_{Q}(q)\right\rangle
$$

where $q \in Q$ and $\alpha_{q} \in T_{q}^{*} Q$. In this equation, the pairing on the right is the natural pairing between $T_{q} Q$ and $T_{q}^{*} Q$, while the pairing on the left is between $\mathfrak{g}$ and $\mathfrak{g}^{*}$. 


\subsubsection{A connection on the reduction bundle}

As shown in Marsden et al. (1990) (see also Marsden, 1992), when the coadjoint isotropy subgroup $G_{\mu}$ is diffeomorphic to $S^{1}$ or $\mathbb{R}$, there is an interesting connection $\gamma$ on the reduction bundle $\pi_{\mu}$ (see Marsden et al. (2000) for a generalization of the construction).

Namely, the $\mathfrak{g}_{\mu} \cong \mathbb{R}$-valued connection one-form is given by

$$
\gamma=\frac{1}{\langle\mu, \zeta\rangle} \theta_{\mu} \otimes \zeta
$$

We explain the notation. As above, $\mu$ is a regular value of the momentum map $\mathbf{J}$ and $P_{\mu}=\mathbf{J}^{-1}(\mu) / G_{\mu}$. Also, $\zeta$ is a generator of the Lie algebra $\mathfrak{g}_{\mu} \cong \mathbb{R}$, and $\theta_{\mu}$ is the canonical one-form on $T^{*} Q$ restricted to $\mathbf{J}^{-1}(\mu)$.

\subsubsection{The connection for vortex rings}

In our problem, $Q=\mathbb{R}^{2}$ with coordinates $\left(X_{1}, X_{2}\right)$ and $G_{\mu}=G=\mathbb{R}$. Choose canonical cotangent coordinates $\left(X_{1}, X_{2}, p_{1}, p_{2}\right)$ for $T^{*} Q$. The phase space is $P=T^{*} Q \backslash \Delta=\left(\mathbb{R}^{2} \times\left(\mathbb{R}^{2}\right)^{*}\right) \backslash \Delta$. Here we identify the dual space $\left(\mathbb{R}^{2}\right)^{*}$ with $\mathbb{R}^{2}$ using the standard Euclidean inner product. Accordingly, we make the identification $\alpha_{q}=\left(p_{1}, p_{2}\right) \in \mathbb{R}^{2}$.

Now consider the group $G=\mathbb{R}$ acting on $Q$ by translations; the group element $g \in \mathbb{R}$ acts on $\left(X_{1}, X_{2}\right)$ to give $\left(X_{1}+g, X_{2}+g\right)$. The infinitesimal generator is given simply by $\xi_{Q}\left(X_{1}, X_{2}\right)=(\xi, \xi)$, where $\xi \in \mathfrak{g}=\mathbb{R}$. The momentum map $\mathbf{J}:\left(\mathbb{R}^{2} \times\left(\mathbb{R}^{2}\right)^{*}\right) \backslash \Delta \rightarrow \mathbb{R}^{*}$, is easily computed using (2.7) to be

$$
\mathbf{J}\left(X_{1}, X_{2}, p_{1}, p_{2}\right)=p_{1}+p_{2}
$$

Thus, the level set $\mathbf{J}^{-1}(\mu)$ is defined by the equation $p_{1}+p_{2}=\mu$ and we can use, for example, $\left(X_{1}, X_{2}, p_{1}\right)$ as coordinates for this level set. The canonical 1 -form on $T^{*} Q$ is $\theta=p_{1} \mathrm{~d} X_{1}+p_{2} \mathrm{~d} X_{2}$. The restriction of $\theta$ to $\mathbf{J}^{-1}(\mu)$ is thus given by

$$
\theta_{\mu}=p_{1} \mathrm{~d} X_{1}+\left(\mu-p_{1}\right) \mathrm{d} X_{2} .
$$

Let $\zeta \in \mathbb{R}, \zeta \neq 0$, be thought of as a generator of the Lie algebra of the translation group. Using the general formula (2.8), we find that the Lie algebra-valued connection one-form $\gamma$ on the bundle $\pi_{\mu}: \mathbf{J}^{-1}(\mu) \rightarrow \mathbf{J}^{-1}(\mu) / G:=P_{\mu}$ is given by

$$
\gamma=\frac{p_{1} \mathrm{~d} X_{1}+p_{2} \mathrm{~d} X_{2}}{p_{1}+p_{2}}=\frac{1}{\mu}\left(p_{1} \mathrm{~d} X_{1}+\left(\mu-p_{1}\right) \mathrm{d} X_{2}\right) .
$$

It should be noted that the above connection is not valid for the zero-momentum case, i.e. when $p_{1}+p_{2}=0$. This is interesting in view of the fascinating experiments by Lim and Nickels (1992) on the head-on collision of two circular vortex rings of equal size and equal but opposite strengths. This configuration corresponds to a zero-momentum case in this canonical model and suggests that it needs to be extended to handle such cases and to maybe offer an explanation for the spectacular instabilities that are triggered by such collisions. 


\subsubsection{The curvature of $\gamma$}

We now compute the curvature of the connection $\gamma$, a two-form on the space $P_{\mu}$. We first recall the general computation using the exterior derivative and the horizontal lift:

$$
\begin{aligned}
\operatorname{curv}(\gamma)(u, v) & =\mathbf{d} \gamma(\operatorname{hor}(u), \operatorname{hor}(v)) \\
& =\frac{1}{\langle\mu, \zeta\rangle} \mathbf{d} \theta_{\mu}(\operatorname{hor}(u), \operatorname{hor}(v)) \otimes \zeta \\
& =-\frac{1}{\langle\mu, \zeta\rangle} i_{\mu}^{*} \Omega(\operatorname{hor}(u), \operatorname{hor}(v)) \otimes \zeta \\
& =-\frac{1}{\langle\mu, \zeta\rangle} \pi_{\mu}^{*} \Omega_{\mu}(\operatorname{hor}(u), \operatorname{hor}(v)) \otimes \zeta \\
& =-\frac{1}{\langle\mu, \zeta\rangle} \Omega_{\mu}(u, v) \otimes \zeta,
\end{aligned}
$$

where $\Omega_{\mu}$ is the reduced symplectic form on $P_{\mu}$ and hor $(u)$ and hor $(v)$ are horizontal lifts of vectors $u, v \in T_{[z]} P_{\mu}$, the tangent space of $P_{\mu}$ at a point $[z]=\pi_{\mu}(z)$ and where the horizontal lift is to the point $z$ (the answer is of course independent of the choice of representative $z$ of the class $[z]$ ).

Using its description in terms of the equation $p_{1}+p_{2}=\mu$, we see that the level set $\mathbf{J}^{-1}(\mu)$ is diffeomorphic to $\left(\mathbb{R}^{2} \times \mathbb{R}\right) \backslash \Delta$. The reduced space $P_{\mu}$ is diffeomorphic to $(\mathbb{R} \times \mathbb{R}) \backslash \Delta_{\mu}$, where $\Delta_{\mu}$ is the single point that $\Delta$ projects to under $\pi_{\mu}$. The phase portrait on $P_{\mu}$ is a function of the parameter values $\Gamma_{1}, \Gamma_{2}, A_{1}$ and $A_{2}$.

In general, for cotangent bundles and reduction by Abelian groups, $P_{\mu} \cong T^{*}(Q / G)$ and the reduced symplectic form is given, by cotangent bundle reduction theory (see, for example, Marsden, 1992), as

$$
\Omega_{\mu}=\Omega_{\mathrm{can}}+\beta_{\mu},
$$

where $\Omega_{\text {can }}$ is the canonical symplectic form and $\beta_{\mu}$ is a "magnetic term", a two-form on the reduced space $Q / G_{\mu}$. Since $Q / G_{\mu}$ is one-dimensional in our problem of two vortex rings, $\beta_{\mu}=0$.

Choosing coordinates $\left(X_{1}, X_{2}, p_{1}\right)$ for $\mathbf{J}^{-1}(\mu)$ and $\left(X_{1}-X_{2}, p_{1}\right)$ for $P_{\mu}$, formula $(2.11)$ gives

$$
\Omega_{\mu}=\mathrm{d}\left(X_{1}-X_{2}\right) \wedge \mathrm{d} p_{1} .
$$

Alternatively, $\Omega_{\mu}$ can be calculated directly from the symplectic reduction theorem (Marsden and Weinstein, 1974) as $\pi_{\mu}^{*} \Omega_{\mu}=i_{\mu}^{*} \Omega$.

\subsubsection{Total phase}

The total phase, $X_{\text {tot }}$, is that group element which measures the total shift in the symmetry group direction at the end of one periodic orbit $c_{\mu}(t) \in P_{\mu}$. In general, $X_{\text {tot }}=X_{\mathrm{d}} \circ X_{\mathrm{g}}$, where $X_{\mathrm{d}}$ is called the dynamic phase, $X_{\mathrm{g}}$ is called the geometric phase and $\circ$ denotes group composition. In this problem:

$$
X_{\text {tot }}=X_{\mathrm{d}}+X_{\mathrm{g}} \text {. }
$$

The total phase is indicated for a typical leapfrogging orbit in Fig. 2. 


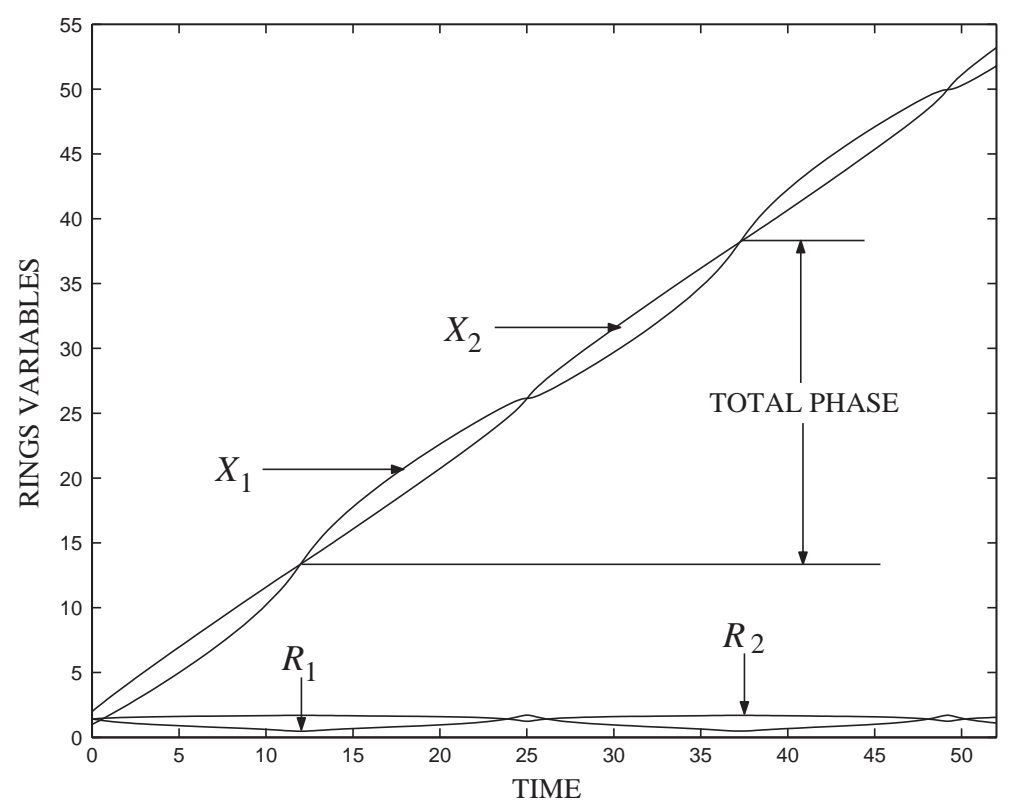

Fig. 2. Dynamic orbits of a leapfrogging pair for the momentum value $\mu=6$ and the following values of the parameters: $\Gamma_{1}=1, \Gamma_{2}=2, A_{1}=A_{2}=0.001$. The variables of the rings shown are the radii, $R_{1}$ and $R_{2}$, and the distances along the axis of symmetry, $X_{1}$ and $X_{2}$. The total phase, which is the total change in $X_{1}$ or $X_{2}$ in one leapfrogging period, is indicated.

\subsubsection{Dynamic phase}

For Abelian groups, the dynamic phase can be explicitly calculated using the exponential map (see Marsden (1992) or Marsden et al. (1990)):

$$
X_{\mathrm{d}}=\exp \int_{0}^{T} \xi(t) \mathrm{d} t
$$

where $\xi(t)$ is a curve in the Lie algebra $\mathfrak{g}$ determined by

$$
\xi(t)=\gamma\left(V_{\mathrm{H}}\left(h_{\mu}(t)\right)\right),
$$

where $h_{\mu}(t)$ is the horizontal lift of the curve $c_{\mu}(t)$ with $h_{\mu}(0)$ being the initial point of the reconstructed trajectory, and $V_{\mathrm{H}}$ is the Hamiltonian vector field on $\mathbf{J}^{-1}(\mu)$ determined uniquely by the given Hamiltonian $H$. The horizontally lifted curve $h_{\mu}(t)$ can be constructed by integrating the following equation:

$$
\frac{\mathrm{d} h_{\mu}}{\mathrm{d} t}=\operatorname{hor}_{h_{\mu}(t)}\left(\frac{\mathrm{d} c_{\mu}}{\mathrm{d} t}\right),
$$

where hor $h_{h_{\mu}(t)}$ means the horizontal lift to the point $h_{\mu}(t)$. In principle, the dynamic phase can then be computed using (2.13) and (2.15).

In this simple example, we can also obtain an integral expression for the dynamic phase without having to solve the differential equation (2.14). To do this, first, note that because the group is 


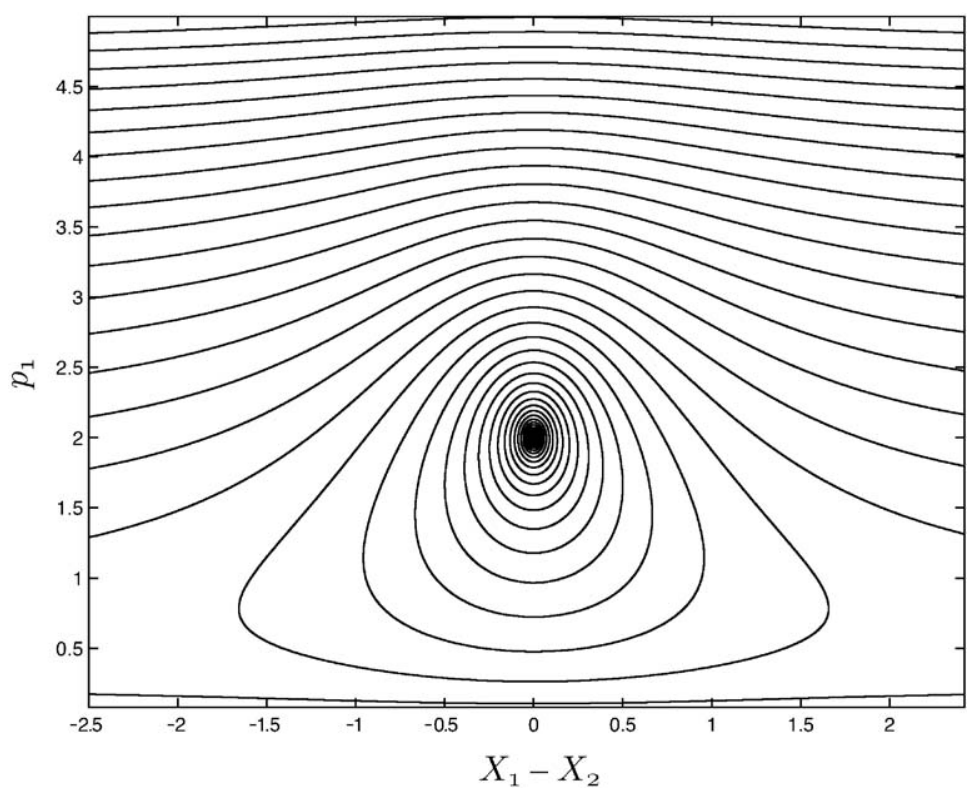

Fig. 3. Phase portrait on the symplectic reduced space $P_{\mu}$ for the momentum value $\mu=6$ and the following values of the parameters: $\Gamma_{1}=1, \Gamma_{2}=2, A_{1}=A_{2}=0.001$. The "center" is not an equilibrium point but is the singular point $\Delta_{\mu}$.

Abelian in our case, the Lie algebra curve in (2.13) can also be obtained as

$$
\xi(t)=\gamma\left(\frac{\mathrm{d} q_{\mu}(t)}{\mathrm{d} t}\right)
$$

where $q_{\mu}(t) \in \mathbf{J}^{-1}(\mu)$ is the reconstructed trajectory which starts from $q_{i}$ and ends at $q_{f}$. From expression (2.10) for $\gamma$, this gives:

$$
\xi(t)=\frac{1}{\mu}\left(p_{1} \frac{\mathrm{d} X_{1}}{\mathrm{~d} t}+\left(\mu-p_{1}\right) \frac{\mathrm{d} X_{2}}{\mathrm{~d} t}\right)
$$

and so the dynamic phase is

$$
X_{d}=\frac{1}{\mu} \int_{0}^{T}\left(p_{1} \frac{\mathrm{d} X_{1}}{\mathrm{~d} t}+\left(\mu-p_{1}\right) \frac{\mathrm{d} X_{2}}{\mathrm{~d} t}\right) \mathrm{d} t .
$$

\subsubsection{The geometric phase}

A typical phase portrait on $P_{\mu}$ is shown in Fig. 3. The closed curves correspond to leapfrogging orbits. The geometric phase, an element of the symmetry group, associated to such a closed curve $c_{\mu} \subset P_{\mu}$ can be expressed as the integral of the negative of the curvature of the connection one-form over the domain $A_{\mu}$ enclosed by $c_{\mu}$. See, for example, Marsden et al. (1990, Corollary 4.2). The geometric phase is therefore

$$
X_{\mathrm{g}}=\exp \left(-\int_{A_{\mu}} \operatorname{curv}(\gamma)\right)=\left(\frac{1}{\mu} \int_{A_{\mu}} \mathrm{d}\left(X_{1}-X_{2}\right) \wedge \mathrm{d} p_{1}\right),
$$


where the exponential map $\exp : \mathfrak{g} \rightarrow G$ in this problem, where $G=\mathbb{R}$ under addition, is just the identity map. Since $\mathrm{d}\left(X_{1}-X_{2}\right) \wedge \mathrm{d} p_{1}$ is the standard volume form on $P_{\mu}$ (also defining an orientation on $P_{\mu}$ ), this clearly shows that

$$
X_{\mathrm{g}}=\left(\frac{\text { Area }_{\mu}}{\mu}\right) \text {, }
$$

where Area $_{\mu}$ is the area enclosed by $c_{\mu}$.

Finally, we can verify that $X_{\text {tot }}=X_{\mathrm{d}}+X_{\mathrm{g}}$. For this, rewrite $X_{\mathrm{g}}$ using Green's theorem in the plane as

$$
X_{\mathrm{g}}=\frac{1}{\mu} \int_{A_{\mu}} \mathrm{d}\left(X_{1}-X_{2}\right) \wedge \mathrm{d} p_{1}=-\frac{1}{\mu} \oint p_{1} \mathrm{~d}\left(X_{1}-X_{2}\right)=-\frac{1}{\mu} \int_{0}^{T} p_{1}\left(\frac{\mathrm{d} X_{1}}{\mathrm{~d} t}-\frac{\mathrm{d} X_{2}}{\mathrm{~d} t}\right) \mathrm{d} t .
$$

One can check that the dynamic trajectories in Fig. 3 are oriented in the counterclockwise sense in the plane with $X_{1}-X_{2}$ and $p_{1}$ as coordinates, so the parametrization of the curves by time gives the correct orientation for the use of Green's theorem. The contour integral in the above is around the closed curve $c_{\mu}(t)$ in $P_{\mu}$. Thus,

$$
X_{\mathrm{d}}+X_{\mathrm{g}}=\int_{0}^{T} \frac{\mathrm{d} X_{2}}{\mathrm{~d} t} \mathrm{~d} t=X_{\mathrm{tot}}
$$

\subsubsection{A metric description of the connection $\gamma$}

As with any connection, $\gamma$ defines a direct sum splitting of the tangent bundle of $\mathbf{J}^{-1}(\mu)$ into horizontal and vertical vectors. We shall show that this splitting is metrically orthogonal with respect to an interesting metric on $\mathbf{J}^{-1}(\mu)$.

In our case, the horizontal and vertical splitting is given as follows. A vector $v_{q} \in T_{q} \mathbf{J}^{-1}(\mu)$ can be written uniquely as

$$
\begin{aligned}
v_{q} & \equiv\left(P \frac{\partial}{\partial p_{1}}, Q \frac{\partial}{\partial X_{1}}, R \frac{\partial}{\partial X_{2}}\right) \\
& =\left(0, K \frac{\partial}{\partial X_{1}}, K \frac{\partial}{\partial X_{2}}\right)+\left(P \frac{\partial}{\partial p_{1}},(Q-K) \frac{\partial}{\partial X_{1}},(R-K) \frac{\partial}{\partial X_{2}}\right) \\
& =\operatorname{ver} v_{q}+\operatorname{hor} v_{q},
\end{aligned}
$$

where

$$
K=\frac{p_{1} Q+\left(\mu-p_{1}\right) R}{\mu} .
$$

Consistent with this claim, it can be checked that

$$
\begin{aligned}
& \mathbf{D} \pi_{\mu}(q)\left(\operatorname{ver} v_{q}\right)=0, \\
& \left(\gamma\left(\operatorname{ver} v_{q}\right)\right)_{\mathbf{J}^{-1}(\mu)}=(0, K, K), \\
& \gamma\left(\operatorname{hor} v_{q}\right)=0,
\end{aligned}
$$

where $\mathbf{D} \pi_{\mu}(q)$ is the derivative of the projection map at $q$. 
Consider the following metric on $T^{*} Q$ :

$$
\langle\langle u, v\rangle\rangle_{\left(X_{1}, X_{2}, p_{1}, p_{2}\right)}=p_{1} u_{1} v_{1}+p_{2} u_{2} v_{2}+u_{3} v_{3}+u_{4} v_{4} .
$$

The restriction of this metric to $\mathbf{J}^{-1}(\mu)$ is

$$
\langle\langle u, v\rangle\rangle_{\left(X_{1}, X_{2}, p_{1}\right)}=p_{1} u_{1} v_{1}+\left(\mu-p_{1}\right) u_{2} v_{2}+u_{3} v_{3} .
$$

One checks that the splitting in (2.16) is metric orthogonal. We mention this as a possible link to the notion of the abstract mechanical connection which is a Lie-algebra valued connection one-form defined on the phase space of a system with symmetry. This subject is explored in detail in the papers of Blaom (2000) and Pekarsky and Marsden (2001).

\section{The interaction of $N$ rings of arbitrary shape}

In this section we show how the symplectic structure on a coadjoint orbit corresponding to a general vorticity distribution in a fluid domain, which was derived in Marsden and Weinstein (1983), gives a symplectic form and a Hamiltonian vector field on the phase space of $N$ interacting filament rings of arbitrary shapes in $\mathbb{R}^{3}$ (see Fig. 4). We also calculate the momentum map associated with the $\mathrm{SE}(3)$ symmetry of this model and show that this is $\mathrm{Ad}^{*}$-equivariant. It should be noted that in this section (and in the next) we choose to model the rings as filaments, as was done in Marsden and Weinstein (1983). We believe that the geometric ideas presented here will still have relevance to more sophisticated ring interaction models which account for core structure.

The Hamiltonian function is still the total kinetic energy of the fluid but becomes unbounded due the well-known singularity in the self-induced velocity field (Saffman, 1992). Desingularization is done by introducing, in the equations of motion, the local induction truncation parameter $c_{j}$ for each ring. This is a (real) truncation parameter that appears in the local induction approximation (Saffman, 1992; Ting and Klein, 1991) for the self-induced velocity field. It represents a choice of the length of the $i$ th ring used to form a finite approximation of the unbounded term in the kinetic energy of the fluid arising due to the singularity.

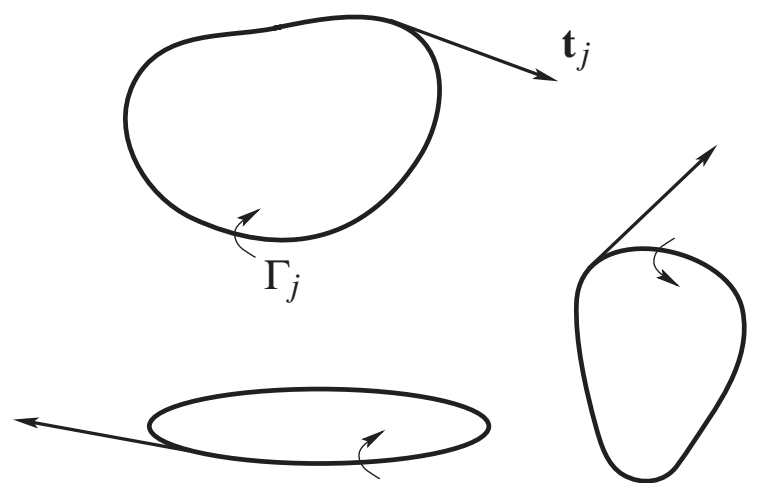

Fig. 4. Interacting vortex filament rings of arbitrary shape. The curved arrows indicate the sense of circulation and the tangential arrows indicate the vorticity direction at the respective points. 


\subsection{The energy expression}

Consider the kinetic energy of a fluid of unit density in $\mathbb{R}^{3}$ :

$$
H=\frac{1}{2} \int\langle\mathbf{A}(\mathbf{x}), \omega(\mathbf{x})\rangle \mathrm{d} V
$$

where the integration is over $\mathbf{x} \in \mathbb{R}^{3}, \mathbf{A}: \mathbb{R}^{3} \rightarrow \mathbb{R}^{3}$ is the vector potential, $\omega: \mathbb{R}^{3} \rightarrow \mathbb{R}^{3}$ is the vorticity, and the pairing $\langle$,$\rangle is the Euclidean inner product on \mathbb{R}^{3}$. The vector potential satisfies

$$
\nabla^{2} \mathbf{A}=-\omega
$$

and inverting this standard Poisson equation gives

$$
\mathbf{A}(\mathbf{x})=\frac{1}{4 \pi} \int \frac{\omega\left(\mathbf{x}^{\prime}\right)}{r^{\prime}} \mathrm{d} V^{\prime}
$$

where $r^{\prime}=\sqrt{\left(x-x^{\prime}\right)^{2}+\left(y-y^{\prime}\right)^{2}+\left(z-z^{\prime}\right)^{2}}$.

Let the vorticity be concentrated along $N$ rings of strengths $\Gamma_{1}, \ldots, \Gamma_{N}$ respectively. As a two-form, the vorticity is represented, following Marsden and Weinstein (1983), as

$$
\omega(x, y, z)=\sum \Gamma_{i} \mathbf{i}_{\mathbf{t}_{i}} \mathrm{~d} x \wedge \mathrm{d} y \wedge \mathrm{d} z \delta_{i}(x, y, z),
$$

where the operator $\mathbf{i}_{\mathbf{t}_{i}}$ denotes the contraction of the three-form along $\mathbf{t}_{i}$, the unit tangent vector field of the $i$ th ring, and $\delta_{i}$ is the delta-function defined with respect to arc-length of the $i$ th ring. The vector potential becomes

$$
\mathbf{A}(\mathbf{x})=\sum \frac{\Gamma_{i}}{4 \pi} \oint_{i} \frac{\mathbf{t}_{i}\left(s_{i}^{\prime}\right) \mathrm{d} s_{i}^{\prime}}{r_{i}^{\prime}}
$$

where $r_{i}^{\prime}=\sqrt{\left(x-x^{\prime}\left(s_{i}^{\prime}\right)\right)^{2}+\left(y-y^{\prime}\left(s_{i}^{\prime}\right)\right)^{2}+\left(z-z^{\prime}\left(s_{i}^{\prime}\right)\right)^{2}}$.

The kinetic energy can then be written as

$$
H=\sum \sum_{j \neq i} \frac{\Gamma_{i} \Gamma_{j}}{8 \pi} \oint_{i} \oint_{j}\left\langle\mathbf{t}_{j}\left(s_{j}^{\prime}\right), \mathbf{t}_{i}\left(s_{i}\right)\right\rangle \frac{\mathrm{d} s_{j}^{\prime}}{r_{j}^{\prime}} \mathrm{d} s_{i}+\sum H_{\mathrm{SI}}^{i},
$$

where $H_{\mathrm{SI}}^{i}$ is the kinetic energy of self-induction of the $i$ th ring. This term is unbounded and we desingularize it as follows using the local induction approximation:

$$
H_{\mathrm{SI}}^{i}=\lim _{c_{i} \rightarrow 0} \frac{\Gamma^{2}}{8 \pi} \oint_{i} \int_{i \pm c_{i}} \frac{\left\langle\mathbf{t}_{i}\left(s_{i}^{\prime}\right), \mathbf{t}_{i}\left(s_{i}\right)\right\rangle \mathrm{d} s_{i}^{\prime}}{\sqrt{\left(x\left(s_{i}\right)-x^{\prime}\left(s_{i}^{\prime}\right)\right)^{2}+\left(y\left(s_{i}\right)-y^{\prime}\left(s_{i}^{\prime}\right)\right)^{2}+\left(z\left(s_{i}\right)-z^{\prime}\left(s_{i}^{\prime}\right)\right)^{2}}} \mathrm{~d} s_{j},
$$

where $i \pm c_{i},\left(c_{i}>0\right)$ indicates that the portion of ring, $s_{i}-c_{i}<s_{i}^{\prime}<s_{i}+c_{i}$, is ignored in evaluating the inner integral. Choosing $s_{i}$ and $s_{i}^{\prime}$ to be arc-length parameters, write

$$
\begin{aligned}
& x^{\prime}\left(s_{i}^{\prime}\right)=x\left(s_{i}\right)+\left(s_{i}^{\prime}-s_{i}\right) \frac{\mathrm{d} x\left(s_{i}\right)}{\mathrm{d} s_{i}}+\cdots, \\
& \mathbf{t}_{i}\left(s_{i}^{\prime}\right)=\mathbf{t}_{i}\left(s_{i}\right)+\left(s_{i}^{\prime}-s_{i}\right) \frac{\mathrm{d} \mathbf{t}_{i}\left(s_{i}\right)}{\mathrm{d} s_{i}}+\cdots
\end{aligned}
$$


and so on. Hence, as $c_{i} \rightarrow 0$,

$$
\begin{aligned}
H_{\mathrm{SI}}^{i} & =\frac{\Gamma^{2}}{8 \pi} \oint_{i} \int_{i \pm c_{i}} \frac{\mathrm{d} s_{i}^{\prime}}{\left|s_{i}^{\prime}-s_{i}\right|} \mathrm{d} s_{i}+O(1) \\
& =\frac{\Gamma^{2}}{8 \pi} \oint_{i}\left(\int_{0}^{s_{i}-c_{i}} \frac{\mathrm{d} s_{i}^{\prime}}{\left|s_{i}^{\prime}-s_{i}\right|}+\int_{s_{i}+c_{i}}^{l_{i}} \frac{\mathrm{d} s_{i}^{\prime}}{\left|s_{i}^{\prime}-s_{i}\right|}\right) \mathrm{d} s_{i}+O(1) \\
& =\frac{\Gamma^{2}}{8 \pi} \oint_{i}\left(-\log \left|s_{i}^{\prime}-s_{i}\right|_{0}^{s_{i}-c_{i}}+\log \left|s_{i}^{\prime}-s_{i}\right|_{s_{i}+c_{i}}^{l_{i}}\right) \mathrm{d} s_{i}+O(1) \\
& =-\frac{\Gamma^{2}}{4 \pi} \oint_{i} \log c_{i} \mathrm{~d} s_{j}+O(1) .
\end{aligned}
$$

The local induction approximation is the choice of a small non-zero value of $c_{i}$ (or the choice of a small non-zero lower bound if $c_{i}$ is allowed to vary in time) at each point of the ring and truncating the above series to leading order:

$$
H_{\mathrm{SI}}^{i} \approx H_{\mathrm{LI}}^{i}
$$

where

$$
H_{\mathrm{LI}}^{i}=-\frac{\Gamma^{2}}{4 \pi} \oint_{i} \log c_{i} \mathrm{~d} s_{i} .
$$

In our model we further assume that each $c_{i}$ is fixed by the initial configuration of the rings and does not vary either with the arc-length $s_{i}$ or with time as the rings evolve. This means, in particular, that we do not look at variations in $c_{i}$ while deriving the equations for the interacting rings. Thus, our final expression for the energy is given as follows:

$$
H=\sum \sum_{j \neq i} \frac{\Gamma_{i} \Gamma_{j}}{8 \pi} \oint_{i} \oint_{j}\left\langle\mathbf{t}_{j}\left(s_{j}^{\prime}\right), \mathbf{t}_{i}\left(s_{i}\right)\right\rangle \frac{\mathrm{d} s_{j}^{\prime}}{r_{j}^{\prime}} \mathrm{d} s_{i}-\sum \frac{\Gamma_{i}^{2}}{4 \pi} \log c_{i} \oint_{i} \mathrm{~d} s_{i} .
$$

\subsection{The symplectic form}

For fixed $\Gamma_{1}, \ldots, \Gamma_{N}$ the set of all such vorticities forms a coadjoint orbit in the dual of the Lie algebra of volume preserving diffeomorphisms of the fluid domain. The symplectic structure on the coadjoint orbit is given by Marsden and Weinstein (1983):

$$
\Omega\left(\mathscr{L}_{u} \omega, \mathscr{L}_{v} \omega\right)=\int \omega(u, v) \mathrm{d} V
$$

where $u(x, y, z), v(x, y, z)$ are divergence-free vector fields on $\mathbb{R}^{3}$ and the Lie derivatives $\mathscr{L}_{u} \omega$ and $\mathscr{L}_{v} \omega$ are tangent vectors to the coadjoint orbit at $\omega \in \mathfrak{g}^{*}$. Since $\mathfrak{g}^{*}$ is a linear space, the tangent vectors can also be identified with vorticity elements and it can be shown (Marsden and Weinstein, 1974) that $\mathscr{L}_{u} \omega \equiv \nabla \times(\omega \times u)$. The vector field $\omega \times u$ is everywhere normal to $\omega$ but is not divergence-free. 
Substituting $\omega$ from (3.1) into (3.4) gives

$$
\Omega=\sum \Gamma_{i} \oint_{i} \mathbf{t}_{i} \cdot\left(u\left(s_{i}\right) \times v\left(s_{i}\right)\right) \mathrm{d} s_{i}=\sum \Gamma_{i} \oint_{i} v\left(s_{i}\right) \cdot\left(\mathbf{t}_{i} \times u\left(s_{i}\right)\right) \mathrm{d} s_{i} .
$$

\subsection{The phase space for $N$ vortex loops}

The vorticities can be identified with the $N$ curves i.e., with images of $N$ maps (modulo re-parametrizations that have the same image) $C_{i}: S^{1} \rightarrow \mathbb{R}^{3}, C_{i}\left(s_{i}\right)=\left(x\left(s_{i}\right), y\left(s_{i}\right), z\left(s_{i}\right)\right), i=1, \ldots, N$ (see Section VI.3 in Arnold and Khesin (1992) for a discussion of this sort of formalism, including knots). The Hamiltonian is regarded as a functional of these curves.

The phase space $P$ for $N$ filaments is thus the space of such curves. The tangent space at any point of the phase space $P$ consists of vector fields along the curves. The symplectic form $\Omega$ given by (3.5) can then be viewed as a symplectic form on $P$. However, $\Omega$ is degenerate and the associated linear map from $T_{p} P$ to $T_{p}^{*} P$ has a non-zero kernel consisting of vector fields tangential to the curves at all points. Indeed, since this kernel is a closed subspace $V_{t} \subset T_{p} P$, one has a direct sum splitting of the tangent space $T_{p} P$ :

$$
\begin{aligned}
& T_{p} P=V_{n} \oplus V_{t}, \\
& v=v_{n}+v_{t},
\end{aligned}
$$

where $v \in T_{p} P, v_{n} \in V_{n}, v_{t} \in V_{t}$, and $V_{n}$ consists of vector fields normal to the curves. The symplectic form $\Omega$, at each $p \in P$, therefore acts on the projection of vectors in $T_{p} P$ on to the subspace $V_{n}$.

\subsection{Computation of functional derivatives}

Let $\varepsilon \delta C_{i}\left(s_{i}\right)=\left(\varepsilon \delta x\left(s_{i}\right), \varepsilon \delta y\left(s_{i}\right), \varepsilon \delta z\left(s_{i}\right)\right)$ denote (small) variations in the curves. Then,

$$
\varepsilon \delta \mathbf{t}_{i}\left(s_{i}\right)=\left(\varepsilon \delta \frac{\mathrm{d} x}{\mathrm{~d} s_{i}}, \varepsilon \delta \frac{\mathrm{d} y}{\mathrm{~d} s_{i}}, \varepsilon \delta \frac{\mathrm{d} z}{\mathrm{~d} s_{i}}\right)=\left(\varepsilon \frac{\mathrm{d} \delta x}{\mathrm{~d} s_{i}}, \varepsilon \frac{\mathrm{d} \delta y}{\mathrm{~d} s_{i}}, \varepsilon \frac{\mathrm{d} \delta z}{\mathrm{~d} s_{i}}\right) .
$$

The functional derivative is defined using the pairing between $T_{p} P$ and $T_{p}^{*} P$ as follows:

$$
\left\langle\delta p, \frac{\delta H}{\delta p}\right\rangle=\sum \oint_{i}\left\langle\delta C_{i}, \frac{\delta H}{\delta C_{i}}\right\rangle \mathrm{d} s_{i}:=\sum \lim _{\varepsilon \rightarrow 0} \frac{1}{\varepsilon}\left(H\left(C_{i}+\varepsilon \delta C_{i}\right)-H\left(C_{i}\right)\right) .
$$

We now calculate

$$
\begin{aligned}
\lim _{\varepsilon \rightarrow 0} \frac{1}{\varepsilon}\left(H\left(C_{i}+\varepsilon \delta C_{i}\right)-H\left(C_{i}\right)\right) \\
=\lim _{\varepsilon \rightarrow 0} \frac{1}{\varepsilon}\left(\Gamma_{i} \sum_{j \neq i} \frac{\Gamma_{j}}{8 \pi} \oint_{i} \oint_{j} \frac{\left\langle\mathbf{t}_{j}\left(s_{j}^{\prime}\right), \mathbf{t}_{i}\left(s_{i}\right)+\varepsilon \delta \mathbf{t}_{i}\right\rangle \mathrm{d} s_{j}^{\prime}}{\sqrt{\left(x_{i}+\varepsilon \delta x_{i}-x_{j}^{\prime}\right)^{2}+\left(y_{i}+\varepsilon \delta y_{i}-y_{j}^{\prime}\right)^{2}+\left(z_{i}+\varepsilon \delta z_{i}-z_{j}^{\prime}\right)^{2}}} \mathrm{~d} s_{i}\right. \\
\quad-\frac{\Gamma_{i}^{2}}{4 \pi} \log c_{i} \oint_{i} \sqrt{\left(\frac{\mathrm{d}\left(x_{i}+\varepsilon \delta x_{i}\right)}{\mathrm{d} s_{i}}\right)^{2}+\left(\frac{\mathrm{d}\left(y_{i}+\varepsilon \delta y_{i}\right)}{\mathrm{d} s_{i}}\right)^{2}+\left(\frac{\mathrm{d}\left(z_{i}+\varepsilon \delta z_{i}\right)}{\mathrm{d} s_{i}}\right)^{2}} \mathrm{~d} s_{i}
\end{aligned}
$$




$$
\begin{aligned}
& -\Gamma_{i} \sum_{j \neq i} \frac{\Gamma_{j}}{8 \pi} \oint_{i} \oint_{j} \frac{\left\langle\mathbf{t}_{j}\left(s_{j}^{\prime}\right), \mathbf{t}_{i}\left(s_{i}\right)\right\rangle \mathrm{d} s_{j}^{\prime}}{\sqrt{\left(x_{i}-x_{j}^{\prime}\right)^{2}+\left(y_{i}-y_{j}^{\prime}\right)^{2}+\left(z_{i}-z_{j}^{\prime}\right)^{2}}} \mathrm{~d} s_{i} \\
& \left.+\frac{\Gamma_{i}^{2}}{4 \pi} \log c_{i} \oint_{i} \sqrt{\left(\frac{\mathrm{d} x_{i}}{\mathrm{~d} s_{i}}\right)^{2}+\left(\frac{\mathrm{d} y_{i}}{\mathrm{~d} s_{i}}\right)^{2}+\left(\frac{\mathrm{d} z_{i}}{\mathrm{~d} s_{i}}\right)^{2}} \mathrm{~d} s_{i}\right) .
\end{aligned}
$$

Ignoring $O\left(\varepsilon^{2}\right)$ and higher order terms we get

$$
\begin{aligned}
\lim _{\varepsilon \rightarrow 0} \frac{1}{\varepsilon}\left(H\left(C_{i}+\varepsilon \delta C_{i}\right)-H\left(C_{i}\right)\right) \\
=\lim _{\varepsilon \rightarrow 0} \frac{1}{\varepsilon}\left(-\Gamma_{i} \sum_{j \neq i} \frac{\Gamma_{j}}{8 \pi}\left(\oint_{i}\left\langle\oint_{j}\left\langle\mathbf{t}_{j}\left(s_{j}^{\prime}\right), \mathbf{t}_{i}\left(s_{i}\right)\right\rangle \frac{\mathbf{R}_{i j}}{r_{i j}^{3}} \mathrm{~d} s_{j}^{\prime}, \varepsilon \delta C_{i}\right\rangle \mathrm{d} s_{i}\right.\right. \\
\left.\left.\left.\quad-\oint_{i}\left\langle\oint_{j} \frac{\mathbf{t}_{j}\left(s_{j}^{\prime}\right)}{r_{i j}} \mathrm{~d} s_{j}^{\prime}, \varepsilon \delta \mathbf{t}_{i}\left(s_{i}\right)\right\rangle \mathrm{d} s_{i}\right)-\frac{\Gamma_{i}^{2}}{4 \pi} \log c_{i} \oint_{i}\left\langle\mathbf{t}_{i}, \varepsilon \delta \mathbf{t}_{i}\right\rangle \mathrm{d} s_{i}\right)\right),
\end{aligned}
$$

where $\mathbf{R}_{i j}$ is the position vector of the point $\left(x_{i}, y_{i}, z_{i}\right)$ with respect to $\left(x_{j}^{\prime}, y_{j}^{\prime}, z_{j}^{\prime}\right)$ and $r_{i j}=\left\|\mathbf{R}_{i j}\right\|$.

Using the elementary identity

$$
\oint f \frac{\mathrm{d} g}{\mathrm{~d} s} \mathrm{~d} s+\oint g \frac{\mathrm{d} f}{\mathrm{~d} s} \mathrm{~d} s=\oint \mathrm{d}(f g)=0
$$

gives

$$
\begin{aligned}
\oint_{i}\left\langle\delta C_{i}, \frac{\delta H}{\delta C_{i}}\right\rangle \mathrm{d} s_{i} & :=\lim _{\varepsilon \rightarrow 0} \frac{1}{\varepsilon}\left(H\left(C_{i}+\varepsilon \delta C_{i}\right)-H\left(C_{i}\right)\right) \\
= & -\Gamma_{i} \sum_{j \neq i} \frac{\Gamma_{j}}{8 \pi}\left(\oint_{i}\left\langle\oint_{j}\left\langle\mathbf{t}_{j}\left(s_{j}^{\prime}\right), \mathbf{t}_{i}\left(s_{i}\right)\right\rangle \frac{\mathbf{R}_{i j}}{r_{i j}^{3}} \mathrm{~d} s_{j}^{\prime}, \delta C_{i}\right\rangle \mathrm{d} s_{i}\right. \\
& \left.+\oint_{i}\left\langle\frac{\mathrm{d}}{\mathrm{d} s_{i}} \oint_{j} \frac{\mathbf{t}_{j}\left(s_{j}^{\prime}\right)}{r_{i j}} \mathrm{~d} s_{j}^{\prime}, \delta C_{i}\right\rangle \mathrm{d} s_{i}\right)+\frac{\Gamma_{i}^{2}}{4 \pi} \log c_{i} \oint_{i}\left\langle\frac{\mathrm{d}}{\mathrm{d} s_{i}} \mathbf{t}_{i}, \delta C_{i}\right\rangle \mathrm{d} s_{i} .
\end{aligned}
$$

Hence,

$$
\begin{aligned}
\frac{\delta H}{\delta C_{i}} & =-\Gamma_{i} \sum_{j \neq i} \frac{\Gamma_{j}}{8 \pi}\left(\oint_{j}\left\langle\mathbf{t}_{j}\left(s_{j}^{\prime}\right), \mathbf{t}_{i}\left(s_{i}\right)\right\rangle \frac{\mathbf{R}_{i j}}{r_{i j}^{3}} \mathrm{~d} s_{j}^{\prime}+\frac{\mathrm{d}}{\mathrm{d} s_{i}} \oint_{j} \frac{\mathbf{t}_{j}\left(s_{j}^{\prime}\right)}{r_{i j}} \mathrm{~d} s_{j}^{\prime}\right)+\frac{\Gamma_{i}^{2} \log c_{i}}{4 \pi} \frac{\mathrm{d} \mathbf{t}_{i}}{\mathrm{~d} s_{i}} \\
& =-\Gamma_{i} \sum_{j \neq i} \frac{\Gamma_{j}}{8 \pi}\left(\oint_{j}\left\langle\mathbf{t}_{j}\left(s_{j}^{\prime}\right), \mathbf{t}_{i}\left(s_{i}\right)\right\rangle \frac{\mathbf{R}_{i j}}{r_{i j}^{3}} \mathrm{~d} s_{j}^{\prime}-\oint_{j} \mathbf{t}_{j}\left(s_{j}^{\prime}\right) \frac{\left\langle\mathbf{R}_{i j}, \mathbf{t}_{i}\left(s_{i}\right)\right\rangle}{r_{i j}^{3}} \mathrm{~d} s_{j}^{\prime}\right)+\frac{\Gamma_{i}^{2} \log c_{i}}{4 \pi} \frac{\mathrm{d} \mathbf{t}_{i}}{\mathrm{~d} s_{i}}
\end{aligned}
$$




$$
\begin{aligned}
& =\Gamma_{i} \sum_{j \neq i} \frac{\Gamma_{j}}{8 \pi}\left(\oint_{j} \mathbf{t}_{i}\left(s_{i}\right) \times \frac{\mathbf{t}_{j}\left(s_{j}^{\prime}\right) \times \mathbf{R}_{i j}}{r_{i j}^{3}} \mathrm{~d} s_{j}^{\prime}\right)-\frac{\Gamma_{i}^{2} \kappa_{i}\left(s_{i}\right) \log c_{i}}{4 \pi}\left(\mathbf{t}_{i} \times \mathbf{b}_{i}\right) \\
& =\Gamma_{i} \sum_{j \neq i} \frac{\Gamma_{j}}{8 \pi}\left(\mathbf{t}_{i}\left(s_{i}\right) \times \oint_{j}\left(\frac{\mathbf{t}_{j}\left(s_{j}^{\prime}\right) \times \mathbf{R}_{i j}}{r_{i j}^{3}}\right)_{n_{i}} \mathrm{~d} s_{j}^{\prime}\right)-\frac{\Gamma_{i}^{2} \kappa_{i}\left(s_{i}\right) \log c_{i}}{4 \pi}\left(\mathbf{t}_{i} \times \mathbf{b}_{i}\right),
\end{aligned}
$$

where we have used the Serret-Frenet equations $\mathrm{d}\left(\mathbf{t}_{i}\left(s_{i}\right)\right) / \mathrm{d} s_{i}=\kappa_{i}\left(s_{i}\right) \mathbf{n}_{i}, \kappa_{i}\left(s_{i}\right)$ is the curvature of the ring, $\mathbf{n}_{i}\left(s_{i}\right)$ and $\mathbf{b}_{i}\left(s_{i}\right)=\mathbf{t}_{i}\left(s_{i}\right) \times \mathbf{n}_{i}\left(s_{i}\right)$ are the unit normal and the unit binormal, respectively, to the ring and, in the last line, the subscript $n_{i}$ denotes the component normal to $C_{i}$. Note that $\delta H / \delta C_{i}$ itself is a vector field normal to $C_{i}$.

\subsection{Computation of the Hamiltonian vector field}

We are now ready to compute the Hamiltonian vector field $X_{H}$ associated to the Hamiltonian $H$ using the general definition $\Omega\left(X_{H}, u\right)=\mathrm{d} H \cdot u$. First of all, write $X_{H}=\left(\partial C_{1} / \partial t, \ldots, \partial C_{N} / \partial t\right)$.

Now compute as follows:

$$
\begin{aligned}
\Omega\left(X_{H}, v_{n}\right) & =\Omega\left(X_{H}, v\right)=\mathrm{DH}\left(C_{1}, \ldots, C_{N}\right) \cdot v \\
& :=\sum \oint_{i}\left\langle v_{i}, \frac{\delta H}{\delta C_{i}}\right\rangle \mathrm{d} s_{i}=\sum \oint_{i}\left\langle\left(v_{i}\right)_{n}, \frac{\delta H}{\delta C_{i}}\right\rangle \mathrm{d} s_{i},
\end{aligned}
$$

for $v_{n} \in V_{n}$. Hence,

$$
\begin{aligned}
& \sum \Gamma_{i} \oint_{i} v_{i} \cdot\left(\mathbf{t}_{i} \times \frac{\partial C_{i}}{\partial t}\right) \mathrm{d} s_{i} \\
& \quad=\sum \Gamma_{i} \oint_{i} v_{i} \cdot\left(\mathbf{t}_{i} \times\left(\sum_{j \neq i} \frac{\Gamma_{j}}{8 \pi} \oint_{j}\left(\frac{\mathbf{t}_{j}\left(s_{j}^{\prime}\right) \times \mathbf{R}_{i j}}{r_{i j}^{3}}\right)_{n_{i}} \mathrm{~d} s_{j}^{\prime}-\frac{\Gamma_{i} \kappa_{i}\left(s_{i}\right) \log c_{i}}{4 \pi} \mathbf{b}_{i}\right)\right) \mathrm{d} s_{i}
\end{aligned}
$$

and therefore

$$
\frac{\partial C_{i}}{\partial t}=\sum_{j \neq i} \frac{\Gamma_{j}}{8 \pi} \oint_{j}\left(\frac{\mathbf{t}_{j}\left(s_{j}^{\prime}\right) \times \mathbf{R}_{i j}}{r_{i j}^{3}}\right)_{n_{i}} \mathrm{~d} s_{j}^{\prime}-\frac{\Gamma_{i} \kappa_{i}\left(s_{i}\right) \log c_{i}}{4 \pi} \mathbf{b}_{i}, \quad i=1, \ldots, N
$$

which are indeed the equations of motion of the rings using the Biot-Savart law and the local induction approximation.

We summarize this calculation as follows:

Proposition 3.1. The filament rings equations of motion (3.8) are Hamiltonian with respect to the Hamiltonian function (3.3) and the symplectic structure (3.5). 


\subsection{The symmetry group}

The action of the group $G=\mathrm{SE}(3)=\left(\mathrm{SO}(3)(S) \mathbb{R}^{3}\right)$ (the semidirect product of rotations and translations $)$ on the $j$ th filament $C_{j}\left(s_{j}\right)=\left(x\left(s_{j}\right), y\left(s_{j}\right), z\left(s_{j}\right)\right)$ is simply

$$
g \cdot C_{j}\left(s_{j}\right)=g \cdot\left(x\left(s_{j}\right), y\left(s_{j}\right), z\left(s_{j}\right)\right), \quad s_{j} \in \operatorname{Dom}\left(C_{j}\right),
$$

where the right-hand side is the usual $\operatorname{SE}(3)$ action on $\mathbb{R}^{3}$. The action on $P$ is then the diagonal action on the $N$ filaments. It is straightforward to check that the symplectic form and the Hamiltonian are invariant under this action of $\operatorname{SE}(3)$.

The infinitesimal generator of the $G$ action on $P$ is calculated in a straightforward way:

$$
\xi_{P}(p):=\left.\frac{\mathrm{d}(g(t) \cdot p)}{\mathrm{d} t}\right|_{t=0},
$$

where $g(t)$ is the one-parameter subgroup such that $g(0)=e$ and $g^{\prime}(t)=\xi \in \mathfrak{g} \equiv \mathfrak{s e}(3)$. Note that (Marsden and Ratiu, 1999, Section 14.9) $\xi$ can be written as the following $4 \times 4$ matrix:

$$
\xi=\left(\begin{array}{cc}
\hat{\xi}_{R} & \xi_{T} \\
0 & 0
\end{array}\right)
$$

where $\hat{\xi}_{R} \in \mathfrak{s o}(3)$ is a skew-symmetric $3 \times 3$ matrix which can be identified uniquely with an element $\xi_{R}$ of $\mathbb{R}^{3}$ via the hat map, and $\xi_{T} \in \mathbb{R}^{3}$. The one-parameter subgroup can be written as

$$
g(t)=I+t \xi+\cdots
$$

(Marsden and Ratiu, 1999, p. 251, ex.(b)). Thus, for $v \in \mathbb{R}^{3}$,

$$
\begin{aligned}
g(t) \cdot v & =I \cdot v+t \hat{\xi}_{R} \cdot v+t \xi_{T}+\cdots \\
& =I \cdot v+t \xi_{R} \times v+t \xi_{T}+\cdots
\end{aligned}
$$

Thus for $p=\left[\left(x\left(s_{1}\right), y\left(s_{1}\right), z\left(s_{1}\right)\right), \ldots,\left(x\left(s_{N}\right), y\left(s_{N}\right), z\left(s_{N}\right)\right]=\left[C_{1}\left(s_{1}\right), \ldots, C_{N}\left(s_{N}\right)\right]\right.$,

$$
\xi_{P}(p)=\left(\xi_{R} \times C_{1}\left(s_{1}\right)+\xi_{T}, \ldots, \xi_{R} \times C_{N}\left(s_{N}\right)+\xi_{T}\right) .
$$

Note that $\xi_{R} \times C_{j}\left(s_{j}\right)$ is a vector field normal to $C_{j}$ but $\xi_{T}$ is not. Recall that the infinitesimal generator of the group action is the Hamiltonian vector field generated by $J(\xi)$, a linear real-valued function on the Lie algebra. Thus,

$$
\sum \Gamma_{i} \oint_{i} v_{i} \cdot\left(\mathbf{t}_{i} \times\left(\xi_{R} \times C_{i}\left(s_{i}\right)+\xi_{T}\right)\right) \mathrm{d} s_{i}=\sum \oint_{i}\left\langle v_{i}, \frac{\delta J(\xi)(p)}{\delta C_{i}}\right\rangle \mathrm{d} s_{i} .
$$

\subsection{The momentum map}

We now calculate the momentum map associated with the SE(3) action on $P$. Recall that (see, for example, Marsden and Ratiu, 1999) for a Lie group action on a Poisson manifold $P$, the momentum map $\mathbf{J}: P \rightarrow \mathfrak{g}^{*}$ given by

$$
\langle\mathbf{J}(p), \xi\rangle=J(\xi)(p) .
$$

Identifying $\mathfrak{s e}(3)$ with $\mathbb{R}^{6}$, the pairing above is the Euclidean inner product on $\mathbb{R}^{6}$. 
From (3.9) we get

$$
\Gamma_{i} \oint_{i}\left(\delta C_{i},\left(\mathbf{t}_{i} \times\left(\xi_{R} \times C_{i}\left(s_{i}\right)+\xi_{T}\right)\right)\right\rangle \mathrm{d} s_{i}=\lim _{\varepsilon \rightarrow 0} \frac{1}{\varepsilon}\left(J(\xi)\left(C_{i}+\varepsilon \delta C_{i}\right)-J(\xi)\left(C_{i}\right)\right) .
$$

It can be checked that the following choice of $J(\xi)$ satisfies the above relation:

$$
J(\xi)=\left\langle\xi_{R},-\frac{1}{2} \sum \Gamma_{i} \oint_{i}\left(\left\langle C_{i}, C_{i}\right\rangle \mathbf{t}_{i}\right) \mathrm{d} s_{i}\right\rangle+\left\langle\xi_{T}, \frac{1}{2} \sum \Gamma_{i} \oint_{i}\left(C_{i} \times \mathbf{t}_{i}\right) \mathrm{d} s_{i}\right\rangle .
$$

To see this, note that

$$
\begin{aligned}
\lim _{\varepsilon \rightarrow 0} \frac{1}{\varepsilon}\left(J(\xi)\left(C_{i}+\varepsilon \delta C_{i}\right)-J(\xi)\left(C_{i}\right)\right)= & \left\langle\xi_{R},-\Gamma_{i} \oint_{i}\left(\left\langle\delta C_{i}, C_{i}\right\rangle \mathbf{t}_{i}+\frac{1}{2}\left\langle C_{i}, C_{i}\right\rangle \delta_{t_{i}}\right) \mathrm{d} s_{i}\right\rangle \\
& +\left\langle\xi_{T}, \frac{1}{2} \Gamma_{i} \oint_{i}\left(\delta C_{i} \times \mathbf{t}_{i}\right) \mathrm{d} s_{i}+\frac{1}{2} \Gamma_{i} \oint_{i}\left(C_{i} \times \delta \mathbf{t}_{i}\right) \mathrm{d} s_{i}\right\rangle .
\end{aligned}
$$

Now use the identities

$$
\oint_{i} \frac{\mathrm{d}}{\mathrm{d} s_{i}}\left(C_{i} \times \delta C_{i}\right) \mathrm{d} s_{i}=0=\oint_{i}\left(C_{i} \times \delta \mathbf{t}_{i}\right) \mathrm{d} s_{i}+\oint_{i}\left(\mathbf{t}_{i} \times \delta C_{i}\right) \mathrm{d} s_{i}
$$

and

$$
\int_{i} \frac{\mathrm{d}}{\mathrm{d} s_{i}}\left\langle C_{i}\left\langle\delta C_{i}, \xi_{R}\right\rangle, C_{i}\right\rangle \mathrm{d} s_{i}=0=\oint_{i} 2\left\langle C_{i}, \mathbf{t}_{i}\right\rangle\left\langle\delta C_{i}, \xi_{R}\right\rangle \mathrm{d} s_{i}+\oint_{i}\left\langle C_{i}, C_{i}\right\rangle\left\langle\delta \mathbf{t}_{i}, \xi_{R}\right\rangle \mathrm{d} s_{i}
$$

to get

$$
\begin{aligned}
\lim _{\varepsilon \rightarrow 0} & \frac{1}{\varepsilon}\left(J(\xi)\left(C_{i}+\varepsilon \delta C_{i}\right)-J(\xi)\left(C_{i}\right)\right) \\
\quad & =-\Gamma_{i} \oint_{i}\left\langle\delta C_{i}, \mathbf{t}_{i} \times\left(C_{i} \times \xi_{R}\right)\right\rangle \mathrm{d} s_{i}+\Gamma_{i} \oint_{i}\left\langle\delta C_{i}, \mathbf{t}_{i} \times \xi_{T}\right\rangle \mathrm{d} s_{i} .
\end{aligned}
$$

We summarize this calculation as follows.

Proposition 3.2. The momentum map for the $\mathrm{SE}(3)$ action on the phase space $P$ of $N$ filament rings is given by

$$
\mathbf{J}(p)=\left(-\frac{1}{2} \sum \Gamma_{i} \oint_{i}\left(\left\langle C_{i}\left(s_{i}\right), C_{i}\left(s_{i}\right)\right\rangle \mathbf{t}_{i}\right) d s_{i}, \frac{1}{2} \sum \Gamma_{i} \oint_{i}\left(C_{i}\left(s_{i}\right) \times \mathbf{t}_{i}\right) \mathrm{d} s_{i}\right) .
$$

In addition, by Noether's theorem the components of the momentum map are conserved quantities, usually referred to as the linear and angular impulse of the fluid. 


\subsection{Equivariance}

As is well known, one has to be careful with equivariance of the momentum map in fluid mechanics. For example, the momentum map associated with the Euclidean group action for the space of $N$ vortices in the plane is not equivariant if the sum of vortex strengths is not zero (see, for example, Adams and Ratiu, 1988; and Marsden and Ratiu, 1999). However in our case, we have

Proposition 3.3. The momentum map (3.11) is $\mathrm{Ad}^{*}$-equivariant.

To prove this, note that the coadjoint action of $(A, a) \in \mathrm{SE}(3)$ on $\mathfrak{s e}(3)^{*}$, after identifying the latter with $\mathbb{R}^{3} \times \mathbb{R}^{3}$, is given as follows (see, for example, Marsden and Ratiu, 1999):

$$
\operatorname{Ad}_{(A, a)^{-1}}^{*}(u, v)=(A u+a \times A v, A v),
$$

where $u$ and $v$ are dual to Lie algebra elements in $\mathfrak{s o}(3)$ and $\mathbb{R}^{3}$, respectively. Writing $\mathbf{J}(p)=(u, v)$, we get:

$$
\begin{aligned}
& \mathbf{J} \cdot((A, a) \cdot p)-\operatorname{Ad}_{(A, a)^{-1}}^{*}(u, v) \\
& =\left(-\frac{1}{2} \sum \Gamma_{i} \oint_{i}\left(\left\langle A C_{i}\left(s_{i}\right)+a, A C_{i}\left(s_{i}\right)+a\right\rangle A \mathbf{t}_{i}\right) \mathrm{d} s_{i}+\frac{1}{2} \sum \Gamma_{i} A \oint_{i}\left(\left\langle C_{i}\left(s_{i}\right), C_{i}\left(s_{i}\right)\right\rangle \mathbf{t}_{i}\right) \mathrm{d} s_{i}\right. \\
& -a \times \frac{1}{2} \sum \Gamma_{i} A \oint_{i}\left(C_{i}\left(s_{i}\right) \times \mathbf{t}_{i}\right) \mathrm{d} s_{i}, \frac{1}{2} \sum \Gamma_{i} \oint_{i}\left(\left(A C_{i}\left(s_{i}\right)+a\right) \times A \mathbf{t}_{i}\right) \mathrm{d} s_{i} \\
& \left.-\frac{1}{2} \sum \Gamma_{i} A \oint_{i}\left(C_{i}\left(s_{i}\right) \times \mathbf{t}_{i}\right) \mathrm{d} s_{i}\right) \\
& =\left(-\sum \Gamma_{i} \oint_{i}\left(\left\langle a, A C_{i}\left(s_{i}\right)\right\rangle A \mathbf{t}_{i}\right) \mathrm{d} s_{i}-a \times \frac{1}{2} \sum \Gamma_{i} A \oint_{i}\left(C_{i}\left(s_{i}\right) \times \mathbf{t}_{i}\right) \mathrm{d} s_{i}, 0\right) \\
& =\left(-\frac{1}{2} \sum \Gamma_{i} \oint_{i}\left(\left\langle a, A C_{i}\left(s_{i}\right)\right\rangle A \mathbf{t}_{i}+\left\langle a, A \mathbf{t}_{i}\right\rangle A C_{i}\right) \mathrm{d} s_{i}, 0\right) \\
& =\left(-\frac{1}{2} \sum \Gamma_{i} \oint_{i} \frac{d}{d s_{i}}\left(\left\langle a, A C_{i}\left(s_{i}\right)\right\rangle A C_{i}\left(s_{i}\right)\right) \mathrm{d} s_{i}, 0\right) \\
& =(0,0) \text {. }
\end{aligned}
$$




\section{Discrete reduction to the case of translating, coaxial, circular rings}

In this final section, we show how the model of $N$ circular filament rings with truncation parameters can be derived from the more general model of Section 3 using discrete symmetries and fixed point sets. It would be nice if one could do a similar construction for the case of thin-cored rings of arbitrary shape, but the extension of the Dyson-Hicks theory to this more general class of rings is not known. Thus, instead, we carry out this program for filament rings.

The reader is referred to Marsden (1992, Chapter 8) where the motivation and methodology of these techniques is discussed in detail.

The model of $N$ circular filament rings with truncation parameters differs from the one used in Section 2 in the self-induced part of the vector field. The equations of motion are:

$$
\begin{aligned}
& \frac{\mathrm{d} X_{i}}{\mathrm{~d} t}=-\frac{\Gamma_{i}}{4 \pi R_{i}}\left(\log c_{i}\right)+\frac{1}{\Gamma_{i}, R_{i}} \frac{\partial U}{\partial R_{i}}, \\
& \frac{\mathrm{d} R_{i}}{\mathrm{~d} t}=-\frac{1}{\Gamma_{i} R_{i}} \frac{\partial U}{\partial X_{i}}, \quad i=1, \ldots, N,
\end{aligned}
$$

where

$$
U=\frac{1}{\pi} \sum_{i=1}^{N} \sum_{j>i}^{N} \Gamma_{i} \Gamma_{j} I_{i j}
$$

and

$$
I_{i j}=\int_{0}^{\pi} \frac{R_{i} R_{j} \cos \theta}{\sqrt{\left(X_{i}-X_{j}\right)^{2}+R_{i}^{2}+R_{j}^{2}-2 R_{i} R_{j} \cos \theta}} \mathrm{d} \theta .
$$

These equations can be cast in the same canonical Hamiltonian form as (2.5) and (2.6) with the same set of canonical variables and symplectic form (2.3), but the Hamiltonian function is different:

$$
H\left(X_{i}, p_{i}\right)=\sum_{i=1}^{N}-\frac{\Gamma_{i}^{3 / 2} p_{i}^{1 / 2}}{2 \pi}\left(\log c_{i}\right)+2 U .
$$

\subsection{The fixing subgroup $\Sigma$}

Fix a line $X$ in $\mathbb{R}^{3}$ passing through the origin. Consider the subgroup $\Sigma \subset \mathrm{SO}(3)$, where $\Sigma$ contains only those elements of $\mathrm{SO}(3)$ whose action is rotation about the line $X$. The action of $\Sigma$ on $P$, denoted by $\sigma_{P}$, is simply the restriction of the larger $\operatorname{SE}(3)$ action.

Consider the action of $\Sigma$ on $\mathrm{SE}(3)$ given by conjugation: $\Phi_{\sigma}(g)=i(\sigma) \cdot g \cdot i\left(\sigma^{-1}\right), \sigma \in \Sigma, g \in \mathrm{SE}(3)$, where $i: \Sigma \rightarrow \mathrm{SE}(3)$ is the inclusion map. The action is a group homomorphism. Further, for $p \in P$,

$$
\begin{aligned}
\Phi_{\sigma}(g) \cdot \sigma \cdot p & =i(\sigma) \cdot g \cdot i\left(\sigma^{-1}\right) \cdot \sigma \cdot p \\
& =\sigma \cdot g \cdot p .
\end{aligned}
$$

Hence, the assumption on the compatibility of the actions (as described in Marsden, 1992) is satisfied. 
In addition, $\mathrm{Ad}^{*}$-equivariance implies, by restricting to elements of $\Sigma$, that the following is also true:

$$
\mathbf{J} \cdot \sigma_{P}=\Phi_{\sigma^{-1}, \mathfrak{g}}^{*} \cdot \mathbf{J}
$$

where $\Phi_{\sigma^{-1}, \mathfrak{g}}^{*}$ is the dual of $\Phi_{\sigma^{-1}, \mathfrak{g}}$, with $\Phi_{\sigma, \mathfrak{g}}: \mathfrak{s e}(3) \rightarrow \mathfrak{s e}(3)$ being the Lie algebra homomorphism induced by the group homomorphism $\Phi_{\sigma}$.

\subsection{The fixed point set}

The fixed point set of the $\Sigma$ action on $P$ is

$$
\begin{aligned}
P_{\Sigma} & =\{p \in P \mid \sigma \cdot p=p \text { for all } \sigma \in \Sigma\} \\
& =\{N \text { circular filaments with the common axis } X\} .
\end{aligned}
$$

The symplectic form on $P_{\Sigma}$ can be obtained from (3.5) by choosing a cylindrical coordinate frame with one axis parallel to the $X$ direction. For normal axisymmetric vector fields $\left(x_{j}^{a}, r_{j}^{a}, 0\right)$ and $\left(x_{j}^{b}, r_{j}^{b}, 0\right)$ we get

$$
\begin{aligned}
\Omega & =\sum \Gamma_{j} \oint_{j}\left\langle(0,0,1),\left(0,0, x_{j}^{a} r_{j}^{b}-x_{j}^{b} r_{j}^{a}\right)\right\rangle R_{j} \mathrm{~d} \theta_{j} \\
& =\sum 2 \pi \Gamma_{j}\left(x_{j}^{a} r_{j}^{b}-x_{j}^{b} r_{j}^{a}\right) R_{j}=\pi \sum \Gamma_{j} \mathrm{~d}\left(x_{j} \wedge r_{j}^{2}\right),
\end{aligned}
$$

which, apart from a multiplicative factor of $\pi$, is the same as (2.3). It should be noted that the Hamiltonian for circular filament rings derived from (3.2) is $\pi$ times the Hamiltonian in (2.4) and the discrete reduction process indeed leads to the same vector field as (2.1) and (2.2). A similar calculation shows that the only non-zero component of the momentum map (3.11) corresponding to symmetry along the $X$-axis, is

$$
\mathbf{J}(p)=\pi \sum \Gamma_{j} R_{j}^{2},
$$

which again, modulo factor $\pi$, is the same as (2.9).

\subsection{The fixed point subgroup}

The fixed point set of the $\Sigma$ action on $\operatorname{SE}(3)$ is

$$
G_{\Sigma}=\left\{g \in \operatorname{SE}(3) \mid \Phi_{\sigma}(g)=g \text { for all } \sigma \in \Sigma\right\} .
$$

However, since $\Sigma \subset \mathrm{SE}(3), \Sigma \subset G_{\Sigma}$, and hence one seeks only elements of $G_{\Sigma} \backslash \Sigma$ since only these have a non-trivial action on $P_{\Sigma}$. Thus $g=(A, \mathbf{a}) \in G_{\Sigma}$ if, for all $\sigma \in \Sigma$ where $\sigma$ can be written as the $3 \times 3$ matrix

$$
\sigma=\left(\begin{array}{ccc}
1 & 0 & 0 \\
0 & \cos \theta & -\sin \theta \\
0 & \sin \theta & \cos \theta
\end{array}\right)
$$


in an orthonormal basis $\left(e_{1}, e_{2}, e_{3}\right)$ where one of the basis vectors is parallel to the $X$ axis, the following holds:

$$
\begin{aligned}
(A, \mathbf{a}) & =(\sigma, 0) \cdot(A, \mathbf{a}) \cdot\left(\sigma^{-1}, 0\right) \\
& =(\sigma, 0) \cdot\left(A \cdot \sigma^{-1}, a\right) \\
& =\left(\sigma \cdot A \cdot \sigma^{-1}, \sigma \cdot a\right) .
\end{aligned}
$$

It is obvious that $\mathbf{a}=\sigma \cdot \mathbf{a}$, for all $\sigma \in \Sigma$ implies $\mathbf{a}=X$. To find the elements that satisfy the other equality, assume there exists $A \notin \Sigma$ such that

$$
A \cdot \mathbf{v}=\sigma \cdot A \cdot \sigma^{-1} \mathbf{v},
$$

for all $\sigma \in \Sigma$ and all $\mathbf{v} \in \mathbb{R}^{3}$. Choose a $\mathbf{v}$ lying on the $X$-axis, so that $\mathbf{v}$ is invariant with respect to the $\Sigma$ action. Then the above equality implies that $A \cdot \mathbf{v}=\sigma \cdot A \cdot \mathbf{v}$ which can be satisfied only if $A \in \Sigma$. Hence,

$$
G_{\Sigma} \backslash \Sigma=X
$$

and we can state that:

Proposition 4.1. The system represented by Eqs. (4.1) and (4.2) is a Hamiltonian subsystem of the Hamiltonian system represented by Eqs. (3.8) in the following sense: it is obtained by discrete reduction with respect to the action of the compact symmetry subgroup $\Sigma \subset \mathrm{SE}(3)$. The phase space $P_{\Sigma}$ of the subsystem is the fixed point set of the action of $\Sigma$ on P. The symmetry group $G_{\Sigma}$ of the subsystem is the fixed point subgroup $G_{\Sigma} / \Sigma$, where $G_{\Sigma}$ is the fixed point set of the action of $\Sigma$ on $\mathrm{SE}(3)$. The Hamiltonian function, symplectic form and momentum map are obtained by simply restricting to $P_{\Sigma}$.

\section{Conclusions and future directions}

Two ideas-geometric phases and discrete reduction-from the geometric theory of reduction of conservative systems with symmetry are applied in this paper to a model of interacting vortex rings in incompressible, inviscid fluid. The authors view this paper as a preliminary step to more sophisticated modeling of ring interactions using ideas from geometric mechanics. In this respect, considerable progress has already been made in the wider context of the averaged Euler equations. See Holm et al. (1998), Oliver and Shkoller (2000) and Marsden and Shkoller (2003) and references therein. The averaged Euler equations may well be the context in which to do more sophisticated ring modeling work since recent results (Holm, 2003) indicate that there is a natural way, consistent with the fundamental variational principles of mechanics, of desingularizing the self-induced velocity field without the introduction of arbitrary parameters as in the local induction approximation. These sorts of models are, as discussed in the paper of Holm, related to all the other interesting models available in the literature; see, for instance Klein and Majda (1993), Klein et al. (1995), Klein and Ting (1992) and references therein.

The motivation for modeling ring interactions comes from many different fluid phenomena of engineering and physics, many of which involve the presence of rigid or deformable boundaries. 
To name a few, the shedding of vortices from the tips of aircraft wings and helicopter blades and the vortices shed by fish (Triantayfyllou and Triantayfyllou, 1995) and birds (Rayner, 1979) during locomotion. A long-term goal of our work is to model rings interacting dynamically with moving solid boundaries. The authors are not aware of any modeling work, even in an inviscid framework (like a vortical extension to Kirchhoff's equations) in this area. Geometric ideas are expected to play key roles in such an area given the well-known geometry of inviscid vortex models (Marsden and Weinstein, 1983) and that of the system of a solid body interacting dynamically with a potential flow field (Leonard and Marsden, 1997). Some work in the 2-D case with point vortices instead of rings has already been done in Shashikanth et al. (2002).

Another future direction suggested by this work and Marsden and Weinstein (1983) is to model the dynamical interaction of $N$ interacting vortex rings of general core structure, with no constraints on the mutual separation distances between rings. Apart from extending the ideas in Sections 3 and 4 in this paper, such a model could also shed light on the phenomenon of vortex stretching which is still not well understood. It has been conjectured, for example in Constantin and Fefferman (1993), that this phenomenon is linked to the lack of a global existence and uniqueness proof for the 3-D Navier-Stokes equations. A related subject on which such a model can have some bearing is that of the development of finite time singularities in solutions of the 3-D Euler and Navier-Stokes equations. For example, in the work by Pelz (1997), it is suggested that a certain configuration of vortex rings with thin cores can display such singular behavior. Of course these questions are also tied up with the issues of the averaged Euler equations since the physical modeling literally by the Euler or Navier-Stokes equations for such singular phenomena is questionable, while working with the averaged equations, for which there is no such singular phenomena, at least with viscosity, is just as reasonable (see Foias et al., 2002; Marsden and Shkoller, 2001).

Finally, we mention that another interesting future direction is that of variational numerical schemes, which automatically preserve at the algorithmic level, the basic fluid dynamical structures, such as vorticity. Of course there are many interesting schemes already available (see, for instance Knio and Klein, 2000), but variational schemes are interesting for long time simulations, as the work of Rowley and Marsden (2002) on planar point vortices shows and it would be interesting to extend this to the case of vortex rings.

\section{Acknowledgements}

The authors would like to thank Darryl Holm and Paul Newton for useful discussions and suggestions. BNS would also like to thank Sergey Pekarsky for some preliminary discussions on cotangent bundle connections.

\section{References}

Adams, M.R., Ratiu, T.S., 1988. The three point vortex problem: commutative and noncommutative integrability. In: Meyer, K. Saari, D. (Eds.), Hamiltonian Dynamical Systems, Contemp. Math. 81, 245-257.

Arnold, V.I., Khesin, B., 1992. Topological methods in hydrodynamics. Ann. Rev. Fluid Mech. 24, $145-166$.

Arnold, V.I., Khesin, B., 1998. Topological Methods in Hydrodynamics, Applied Mathematical Sciences 125, Springer, Berlin. 
Blaom, A.D., 2000. Reconstruction phases via Poisson reduction. Differential Geom. Appl. 12 (3), $231-252$.

Constantin, P., Fefferman, C., 1993. Direction of vorticity and the problem of global regularity of the Navier-Stokes equations. Indiana Univ. Math. J. 42 (3), 775-789.

Dyson, F., 1893. The potential of an anchor ring-Pt. II. Philos. Trans. Roy. Soc. London Ser. A $184,1041-1106$.

Foias, C., Holm, D.D., Titi, E.S., 2002. The three dimensional viscous Camassa-Holm equations and their relation to the Navier-Stokes equations and turbulence theory. Dyn. Differential Equations 14, 1-36.

Gurzhii, A.A., Konstantinov, M.-Yu., Meleshko, V.V., 1988. Interaction of coaxial vortex rings in an ideal fluid. Fluid Dynam. 23, 224-229. Consultants Bureau, New York (translated from Izv. Akad. Nauk SSSR Mekh. Zhidk. Gaza 1988, OVYR, 2, 78-84 (in Russian)).

Hicks, W.M., 1922. On the mutual threading of vortex rings. Proc. Roy. Soc. London Ser. A 102, $111-131$.

Holm, D., 2003. Rasetti-Regge Dirac bracket formulation of Lagrangian fluid dynamics on vortex filaments. Math. Comput. Simulation 62, 53-63.

Holm, D.D., Marsden, J.E., Ratiu, T.S., 1998. The Euler-Poincaré equations and semidirect products with applications to continuum theories. Adv. Math. 137, 1-8.

Klein, R., Majda, A.J., 1993. An asymptotic theory for the nonlinear instability of antiparallel pairs of vortex filaments. Phys. Fluids A 5, 369-379.

Klein, R., Ting, L., 1992. Vortex filament with axial core structure variation. Appl. Math. Lett. 5, 99-103.

Klein, R., Majda, A.J., Damodaran, K., 1995. Simplified equations for the interaction of nearly parallel vortex filaments. J. Fluid Mech. 288, 201-248.

Knio, O.M., Klein, R., 2000. Improved thin-tube models for slender vortex simulations. J. Comput. Phys. 163 , 68-82.

Konstantinov, M.Yu., 1994. Chaotic phenomena in the interaction of vortex rings. Phys. Fluids 6, $1752-1767$.

Lamb, H., 1932. Hydrodynamics, 6th Edition. Dover, New York.

Langer, J., Perline, R., 1991. Poisson geometry of the filament equation. J. Nonlinear Sci. 1, 71-94.

Leonard, N.E., Marsden, J.E., 1997. Stability and drift of underwater vehicle dynamics: mechanical systems with rigid motion symmetry. Physica D 105, 130-162.

Lim, T.T., 1997. http://www.eng.nus.edu.sg/mpelimtt/TT_LIM.htm.

Lim, T.T., Nickels, T.B., 1992. Instability and reconnection in the head-on collision of two vortex rings. Nature 357, 225-227.

Love, A.E.H., 1894. On the motion of paired vortices with a common axis. Proc. London Math. Soc. 25, $185-194$.

Marsden, J.E., 1992. Lectures on Mechanics, London Mathematical Society Lecture Note Series, Vol. 174. Cambridge University Press, Cambridge.

Marsden, J.E., Montgomery, R., Ratiu, T.S., 1990. Reduction, Symmetry and Phases in Mechanics, Memoirs, Vol. 436. American Mathematical Society, Providence, RI.

Marsden, J.E., Ratiu, T.S., 1999. Introduction to Mechanics and Symmetry, Texts in Applied Mathematics, 2nd Edition. Vol. 17, Springer, Berlin, 1999.

Marsden, J.E., Ratiu, T.S., Scheurle, J., 2000. Reduction theory and the Lagrange-Routh equations. J. Math. Phys. 41, 3379-3429.

Marsden, J.E., Shkoller, S., 2001. Global well-posedness of the LANS- $\alpha$ equations. Proc. Roy. Soc. London 359, 1449-1468.

Marsden, J.E., Shkoller, S., 2003. The anisotropic averaged Euler and Navier-Stokes equations. Arch. Rat. Mech. An. $166,27-46$.

Marsden, J.E., Weinstein, A., 1974. Reduction of symplectic manifolds with symmetry. Rep. Math. Phys. 5, $121-130$.

Marsden, J.E., Weinstein, A., 1983. Coadjoint orbits, vortices and Clebsch variables for incompressible fluids. Physica D 7, 305-323.

Oliver, M., Shkoller, S., 2000. The vortex blob method as a second-grade non-Newtonian fluid; E-print, http://xyz.lanl.gov/abs/math.AP/9910088/.

Pekarsky, S., Marsden, J.E., 2001. Abstract mechanical connection and abelian reconstruction for almost Kähler manifolds. J. Appl. Math. 1, 1-28.

Pelz, R., 1997. Locally self-similar finite time collapse in a high-symmetry vortex filament model. Phys. Rev. E 55, $1617-1626$.

Rayner, J.M.V., 1979. A vortex theory of animal flight. Part 2. J. Fluid Mech. 91, 731-763.

Rowley, C.W., Marsden, J.E., 2002. Variational integrators for point vortices. Proc. CDC 40, 1521-1527. 
Saffman, P.G., 1992. Vortex Dynamics. Cambridge Monographs on Mechanics and Applied Mathematics. Cambridge University Press, Cambridge.

Shashikanth, B.N., Marsden, J.E., Burdick, J.W., Kelly, S.D., 2002. The Hamiltonian structure of a 2-D rigid circular cylinder interacting dynamically with $N$ point vortices. Phys. Fluids 14 (3), 1214-1227.

Shashikanth, B.N., Newton, P., 1998. Vortex motion and the geometric phase. Part I. Basic configurations and asymptotics. J. Nonlinear Sci. 8, 183-214.

Ting, L., Klein, R., 1991. Viscous Vortical Flows Lecture Notes in Physics, Vol. 374. Springer, Berlin. Triantayfyllou, M.S., Triantayfyllou, G.S., 1995. An efficient swimming machine. Sci. Am. 272, 64-70. 